\title{
HEMANGIOMA BUCAL EM CRIANÇAS
}

\section{ORAL HEMANGIOMA IN CHILDREN}

\author{
Gisele Fernandes Dias ${ }^{1}$, Luis Henrique Gil França ${ }^{2}$, Fabian Calixto Fraiz ${ }^{3}$, Denise \\ Stadler Wambier ${ }^{4}$, Vitoldo Antonio Kozlowski Jr. ${ }^{5}$, José Miguel Amenabar Céspedes ${ }^{6}$
}

${ }^{1}$ Especialista em Odontopediatria e Saúde Coletiva pela UFPR, Mestranda em Odontologia, Área de Concentração Prevenção pela UEPG. E-mail: < giodonto@hotmail.com>

${ }^{2}$ Doutor em Cirurgia. Departamento de Medicina da UEPG. E-mail: <luishgf@hotmail. com>

${ }^{3}$ Doutor em Odontopediatria. Professor Adjunto do Departamento de Odontologia da UFPR. Faculdade de Odontologia - Universidade Federal do Paraná (UFPR). E-mail: <fraiz@ufpr. br>

${ }^{4}$ Doutora em Odontopediatria. Professora do Curso de Pós-Graduação em Odontologia da UEPG. <dswambier@uepg.br>

${ }^{5}$ Doutor em Odontologia. Professor do Curso de Pós-Graduação em Odontologia da UEPG. <vakozlowskijr@uepg.br>

${ }^{6}$ Doutor em Odontologia. Professor Adjunto do Departamento de Odontologia da UFPR

Data de recebimento: $15 / 05 / 2013$

Data da aprovação: 08/06/2013

\section{RESUMO}

O hemangioma é o tumor vascular benigno mais frequente em crianças, com características peculiares. Alguns fatores de risco são considerados relevantes, como a prematuridade, baixo peso ao nascer, sexo feminino, multiparidade e idade materna avançada. O diagnóstico é realizado por meio da anamnese, avaliação clínica e exames complementares, como: ressonância magnética, tomografia computadorizada, ultrassom e métodos angiográficos. Alguns hemangiomas podem apresentar comprometimento funcional ou complicações, como ulcerações, infecções, sangramentos e complicações sistêmicas durante sua evolução. A história natural do hemangioma é dividida em três fases bem caracterizadas: a fase inicial de crescimento, denominada fase proliferativa, seguida de uma fase de regressão espontânea, ou fase involutiva, e uma terceira fase de equilíbrio final, ou fase involuída. Os hemangiomas podem ser classificados em cutâneos ou viscerais, e as indicações relativas de tratamento estão consideradas de acordo com a localização, profundidade, extensão e dimensão do tumor. As opções de tratamento disponíveis são: a cirurgia, infiltrações locais com agentes esclerosantes, tratamento clínico com corticosteroides, alfa-interferon e betabloqueadores. Este trabalho tem como objetivo uma revisão detalhada sobre hemangioma bucal em crianças e, também, uma análise dos tratamentos disponíveis para esta doença.

Palavras- chave: Hemangioma. Vasos sanguíneos. Criança.

\begin{abstract}
Hemangioma is the most common benign vascular tumor that occurs in children with particular characteristics. The following risk factors were considered relevant prematurity, low birth weight, female sex, advanced maternal age and multiparity. The diagnosis is made based on patients' history, clinical evaluation and laboratory tests such as MRI, CT, ultrasound and angiographic methods. Some hemangiomas may show
\end{abstract}


functional impairment or complications such as ulcers, infections, bleeding and systemic complications during their evolution. The natural history of the hemangioma is divided into three well-characterized phases: the initial growth phase, called proliferative phase followed by a phase of spontaneous regression or involutional stage and a third stage or final equilibrium phase. Hemangiomas can be classified into cutaneous or visceral and the indications of treatment are considered according to the location, depth, length and size of the tumor. Available treatment options are: surgery, infiltration with local sclerosant agents, clinical treatment with corticosteroids, interferon alpha and betablockers. This study aims at a detailed review of oral hemangioma in children and also an analysis of the available treatments for this disease.

Keywords: Hemangioma. Blood vessels. Child.

\section{Introdução}

Hemangioma oral é um tumor benigno de células endoteliais e quando acomete crianças é necessária uma abordagem clínica precoce com a finalidade de evitar traumas psicossociais e deformidades faciais. É uma entidade que, embora rara, acomete principalmente crianças de baixo peso, prematuras, de tez clara, com predileção pelo sexo feminino e tendência familiar (ETHUMANDAM, 2006).

A localização mais prevalente é em palato, pele, gengiva e, quando intraósseos, podem acometer o complexo crânio-facial. Sua história natural não é bem definida por serem desconhecidos os mecanismos embriológicos de formação e também pela dificuldade na detecção precoce desta doença por métodos de imagem. Embora os hemangiomas sejam assintomáticos, complicações como ulcerações, infecções secundárias, ruptura e hemorragia grave podem ocorrer, com risco de óbito do paciente (BOYE, 2009).

Atualmente, com o crescente conhecimento a respeito da lesão, ampliação do acesso aos serviços de saúde bucal pela população e aumento da preocupação estética, a abordagem da lesão tende a se tornar precoce. Sendo assim, o Odontopediatra necessita de conhecimentos atualizados sobre os hemangiomas, pois é crescente a abordagem odontológica na primeira infância e o cirurgião-dentista muitas vezes é o primeiro profissional a entrar em contato com essa entidade.

Os conhecimentos atuais sobre os hemangiomas são ferramentas fundamentais para uma abordagem integral do paciente, com bom senso profissional para encaminhar ou trabalhar em equipe multidisciplinar. Os avanços no tratamento a partir da utilização de substâncias esclerosantes, excisão cirúrgica, embolização associada ao procedimento cirúrgico, uso de laser de luz pulsada, bem como uso de esteroides por aplicação local ou via sistêmica, interferon alfa e recentemente betabloqueadores tornaram alta a taxa de resolução da lesão (ETHUMANDAM, 2006; BOYE, 2009). Este trabalho tem por objetivo realizar revisão da literatura recente sobre hemangiomas, bem como atualizar conhecimentos e orientar condutas a respeito da lesão.

\section{Conceito, Classificação E Prevalência}

Os hemangiomas são divididos em congênitos e presentes ao nascimento. Os hemangiomas congênitos são subdivididos em: rapidamente involutivos, não-evolutivos e progressivos. Os hemangiomas faciais têm predileção por distribuição segmentada e por regiões embriologicamente fusionadas e estão descritos de acordo com a profundidade da lesão em: superficiais, profundos e compostos. Histologicamente são classificados em: capilares, cavernoso e composto, decorrentes do "turnover" das células epiteliais, oriundos do processo de renovação e substituição de células que já não exercem suas atividades corretamente. A forma sistêmica difusa está relacionada à Síndrome de Sturge-Weber (MULLIKEN, 1982). Aalst e colaboradores (2003) afirmam que as lesões vasculares pediátricas são divididas em malformações vasculares e hemangiomas. As malformações vasculares são agrupadas de acordo com o tipo de vaso dominante (capilares, veias, artérias e tecidos linfáticos) e características da superfície e são consideradas erros da morfogênese da vasculatura. As malformações vasculares não regridem e requerem tratamento com embolização e ressecção cirúrgica.

Os hemangiomas tiveram seu conceito e classificação modificados ao longo dos anos. Por 
muito tempo não houve uma classificação diagnóstica aceita internacionalmente, o que dificultou a criação de condutas, bem como comparações entre o manejo da lesão. Segundo Gontijo e colaboradores (2003), o histórico da classificação clínica dos hemangiomas teve início com Virchow, em 1863, com base no quadro microscópico da lesão. As nomenclaturas e o conhecimento da etiologia das anomalias vasculares começaram a ser esclarecidas em 1982, com os estudos de Mulliken e Glowacki (1982), os quais fundamentaram a classificação das lesões vasculares baseados em critérios histopatológicos, história clínica e comportamento da lesão, dividindo-as em malformações vasculares e hemangiomas. Desta forma, os autores propuseram uma classificação das lesões vasculares cutâneas (Tabela 1), a qual, atualmente, representa a classificação padrão das anomalias vasculares em crianças. O objetivo foi estabelecer uma linguagem comum aos diferentes especialistas envolvidos no manejo da lesão. De acordo com a classificação, as anomalias vasculares dividem-se em duas categorias: tumores vasculares, dentre eles os hemangiomas, que são os mais comuns, e malformações vasculares (MULLIKEN, 1982)

Tabela 1 - Classificação das lesões vasculares segundo Mulliken e Glowacki (1982)

\begin{tabular}{c|c}
\hline HEMANGIOMAS & MALFORMAÇÕES \\
\hline Apresentam fase proliferativa e involutiva & $\begin{array}{c}\text { Malformações capilares, venosas, arteriais, linfáticas, e fístulas sem fase pro- } \\
\text { liferativa e involutiva }\end{array}$ \\
\hline Proliferação das células endoteliais & Ciclo das células endoteliais normal \\
\hline $40 \%$ presentes ao nascimento, usualmente como uma mancha vermelha & Crescem proporcionalmente à criança \\
\hline Crescimento pós-natal rápido e involução lenta & Razão sexo feminino:masculino - $1: 1$ \\
\hline Razão sexo feminino:masculino $-5: 1$ & \\
\hline
\end{tabular}

Em 1996, foi adotada pela Sociedade Internacional para o Estudo das Anomalias Vasculares (ISSVA) a classificação - tumores e malformações vasculares - com a finalidade de simplificar a abordagem terapêutica, uniformizar a nomenclatura, atualizar conceitos clínicos e de imagens e também para definir estratégias para o tratamento adequado (ERNEMANN, 2010). Os tumores vasculares incluem: hemangioma da infầncia, hemangioma congênito rapidamente involutivo, hemangioma congênito não-involutivo, granuloma piogênico, hemangioendotelioma Kaposiforme e angioma em tufos (ERNEMANN, 2010).

Em relação ao estágio da lesão são divididos em fase proliferativa e fase involutiva. A fase proliferativa é vista somente em crianças, sendo o período de crescimento restrito aos primeiros anos de vida. Em relação ao crescimento da lesão, os hemangiomas apresentam um ciclo de proliferação, estabilização e involução de forma espontânea, ao passo que as malformações vasculares não (ERNEMANN, 2010). O hemangioma da infância é considerado uma neoplasia vascular benigna por proliferação de células endoteliais. Podem ser classificados histologicamente em superficiais (derme papilar), profundos (derme reticular profunda e tecido celular subcutâneo) e mistos. As lesões superficiais são de coloração vinho tinto e as profundas de coloração azulada de pele suprajacente normal, podendo apresentar-se únicas ou múltiplas (ERNEMANN, 2010).

Ray e Matthew (2009) com o propósito de orientar cirurgiões-dentistas a estabelecer correto diagnóstico e tratamento das anormalidades vasculares, baseados na natureza da lesão, afirmam que a distinção entre as malformações vasculares deve estar baseada na história clínica da lesão, achados histológicos e citoquímicos, profundidade da lesão e características de fluxo (RAY, 2009).

Os hemangiomas são os tumores vasculares benignos mais frequentes dentre todas as lesões tumorais da criança; contudo, a lesão pode aparecer em qualquer idade, com frequência considerável em indivíduos idosos. A prevalência dos hemangiomas em crianças incide em torno de 2 a $3 \%$ e cerca de $10 \%$ em infantes prematuros e de baixo peso (ERNEMANN, 2010).

\section{Fatores De Risco, História Natural}

\section{E Quadro Clínico}

Os fatores de risco para o surgimento dos hemangiomas incluem: baixo peso ao nascer, prema- 
turidade, pessoas de pele clara, tendência familiar e predileção pelo sexo feminino. Os fatores de risco adicionais relatados para os hemangiomas incluem: a prematuridade, sexo feminino, etnia caucasiana, gestação múltipla, idade materna avançada e anormalidades placentárias, dentre estas a placenta prévia (METRY, 2000; ERNEMANN, 2010).

Os hemangiomas têm crescimento inicial rápido de células endoteliais e subsequente involução lenta. A sua história natural é dividida em três fases bem características: primeiro a fase inicial ou proliferativa, platô e involutiva ou fase de equilíbrio final (ERNEMANN, 2010). A fase proliferativa é mais pronunciada nos primeiros 3 a 6 meses de vida e alcança dimensões máximas em torno dos 9 aos 12 meses de idade, sendo que as dimensões são consideráveis em proporção ao crescimento da criança e, dependendo da localização, pode causar comprometimento estético, funcional e psíquico. A fase de involução não significa obrigatoriamente retorno à normalidade, podendo resultar em sequelas, como tumor residual, cicatrizes, telangiectasias, hipo ou hipercromia cutânea e irregularidades locais (RAY, 2009; ERNEMANN, 2010).

De acordo com PAGNONCELLI, (1994), os hemangiomas orais apresentam-se como lesões planas da mucosa, com tamanho e formas variáveis, de coloração vermelho azulada. Podem apresentar localização intraóssea, sendo denominados de nevo flâmeo, com imagem radiográfica sugestiva de aspecto de favos de mel. A localização preferencial dos hemangiomas é a cabeça e pescoço, mas pode acometer o tronco e as extremidades. Na face podem estar localizados em pálpebras, lábio inferior e em boca. A presença da lesão em lábios inferiores acarreta salivação excessiva, transtornos fonéticos, estéticos e de deglutição. Quando intrabucal acomete preferencialmente lábios e mucosa jugal. A maioria dos hemangiomas permanece bem circunscrito, sendo que a lesão pode variar de milímetros a centímetros (ELIAS, 2004; ROCHA, 2000; SILVA, 2000; TRUJILLO, 2008). A aparência clínica é variável, sendo a coloração dependente da profundidade da lesão: quanto mais superficiais a coloração é de vermelho vivo (figura 1), alternando para azul com o aumento da profundidade da lesão (figura 2). A ocorrência de hemangiomas intraósseos gera dificuldades para o diagnóstico diferencial com outras lesões maxilares. O crescimento da lesão é de aproximadamente $90 \%$ próximo ao término do primeiro mês de vida, sendo possível observar locais de involução e crescimento proliferativo, concomitantes na lesão. Os hemangiomas são provenientes de um desequilíbrio na angiogênese no primeiro trimestre de gravidez ou por alteração gênica, o que se confirma pela existência de irmão afetados e incidência alta em alguns grupos familiares (ELIAS, 2004; ROCHA, 2000; SILVA, 2000; TRUJILLO, 2008). .

Figura 1 - Hemangioma superficial localizado na língua.

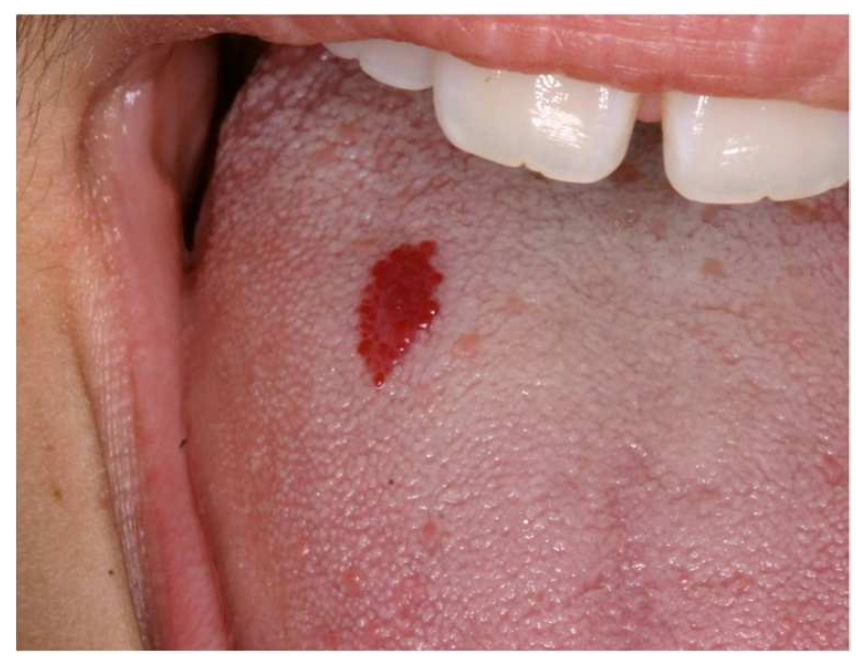

Figura 2 - Hemangioma bucal (lesão mais profunda).

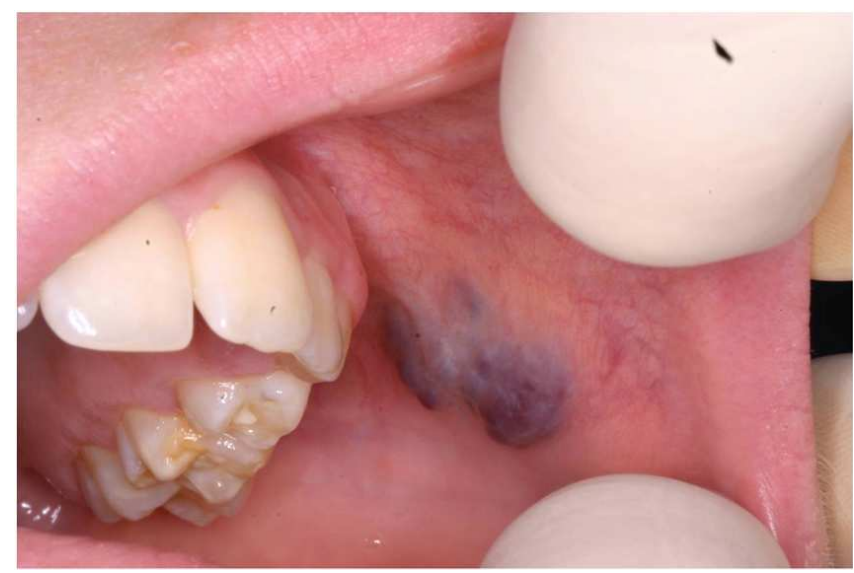

Dentre as complicações decorrentes do hemangioma são destacadas: a ulceração, insuficiência cardíaca congestiva, hipotiroidismo, alteração da visão, comprometimento da respiração, audição e desfiguração. A ulceração é frequente e comum na fase de proliferação rápida, em que gera dor e desconforto, de acordo com a localização da lesão. É comum a presença de infecção secundária em locais ulcerados, geralmente restritos à pele, 
e que pode contribuir para surgimento de quadros de erisipela, celulite e septicemia. A presença de hemangiomas de glândula parótida pode gerar obstrução de conduto auditivo externo, o que acarreta complicações, como redução da condução auditiva, que altera o desenvolvimento da linguagem e gera otites de repetição. Dependendo da localização do tumor, podem ocorrer complicações decorrentes, como bloqueio de vias aéreas, principalmente em região subglótica. Devido à importância clínica do hemangioma, o conhecimento da lesão pelo cirurgião-dentista pode prevenir hemorragias durante as intervenções clínicas invasivas, que podem até pôr em risco a vida do paciente (ROCHA, 2000; ETHUMANDAM, 2006).

O principal diagnóstico diferencial dos hemangiomas é a malformação vascular. TRUJILLO 2008, ao diferenciar hemangiomas das malformações vasculares, ressalta que a história clínica da lesão é fundamental para o diagnóstico. Os hemangiomas podem estar ou não presentes ao nascimento, ao passo que as malformações sempre estão. As malformações vasculares representam lesões congênitas compostas de circuitos vasculares displásicos, sem proliferação endotelial ou regressão espontânea. As malformações vasculares congênitas geralmente estão presentes ao nascimento e crescem proporcionalmente com a criança (TRUJILLO, 2008). Entre outras doenças, temos: o granuloma piogênico, sarcoma de Kaposi, hiperplasia inflamatória gengival, epulis granulomatosa, varicocele, telangiectasia e carcinoma de células escamosas (ELIAS, 2004). A orientação sobre o manejo da lesão reside em detecção de lesões iniciais e em alguns casos a biópsia (ELIAS, 2004; TRUJILLO, 2008).

\section{Exames Complementares}

O uso combinado de modalidades de imagem auxilia na obtenção das informações necessárias, como tamanho, localização, extensão anatômica, anatomia vascular e proximidade de estruturas vitais. Os exames incluem a vitropressão, punção, biópsia, citologia esfoliativa, exames radiográficos, exame ultrassonográfico, ressonância magnética de imagem, tomografia computadorizada e angiografia (METRY, 2000; AALST, 2003; BOYLE, 2009; RAY, 2009). Os exames complementares visam caracterizar, visuali- zar a extensão e profundidade da lesão, o que definirá a abordagem terapêutica. Em relação ao diagnóstico está indicada a vitropressão, sinonímia de diascopia, que confirma o diagnóstico ao apresentar alteração da coloração sugestivo de empalidecimento. O histórico clínico e a vitropressão são métodos soberanos no diagnóstico desse tumor benigno (METRY, 2000; AALST, 2003; BOYLE, 2009; RAY, 2009).

\section{Tratamento}

Embora existam várias opções terapêuticas disponíveis, os hemangiomas intraorais não causam grandes transtornos aos pacientes, salvo em áreas com risco de trauma. Essas áreas podem acarretar hemorragias de grande porte, podendo ocorrer em situações do cotidiano do paciente, como em mordidas involuntárias, traumatismos e perfurações acidentais durante o tratamento odontológico (METRY, 2000; ETHUMANDAM, 2006; BOYE, 2009). SILVA e colaboradores (2000) consideram a indicação do tratamento de hemangiomas em crianças quando a lesão apresentar um importante comprometimento estético ou funcional, todavia a literatura recomenda aguardar a involução espontânea da lesão. Della Nina e colaboradores (2006), em estudo retrospectivo de dez anos sobre alterações vasculares, revelam que diante dos dados epidemiológicos obtidos, em comparação com a literatura mundial no que trata do manejo dos hemangiomas, a conduta expectante alicerçada na tendência natural à regressão da lesão foi a mais comumente encontrada.

A abordagem terapêutica está voltada para os riscos de comprometimento funcional, seja pelas dimensões ou complicações evolutivas da lesão (ETHUMANDAM, 2006). Embora em alguns casos seja recomendada a conduta expectante, quando lesões de pequenas dimensões e sem implicações estéticas, o profissional deve sempre realizar documentação fotográfica evolutiva e, também, obter assinatura de consentimento informado dos pais, com a finalidade de prevenir potenciais processos judiciais (PAGNONCELLI, 1994; METRY, 2000; ROCHA, 2000; TRUJILLO, 2008; BOYE, 2009). O tratamento é indicado em situações específicas, adequados ao volume, extensão, tempo de evolução e localização da lesão, alinhado ao acompanhamento clínico posterior à erradicação da lesão. As situações 
que requerem intervenção precoce dos hemangiomas incluem a proximidade de regiões altamente vascularizadas, obstrução ou compressão de vias aéreas e do canal auditivo, além de coagulopatias e insuficiência cardíaca congestiva causadas por lesões de grande volume (PAGNONCELLI, 1994; METRY, 2000; ROCHA, 2000; ETHUMANDAM, 2006; TRUJILLO, 2008; BOYE, 2009; RAY, 2009). .

O propósito de o tratamento iniciar na infância tem por objetivo erradicar completamente a lesão, diminuir a proliferação e evitar alterações faciais. Os autores orientam a negligência "benigna" em lesões esteticamente aceitáveis, com consentimento familiar e acompanhamento profissional até os 3-4 anos de idade. Nessa faixa etária, a lesão pode se tornar aparente ou não involuir. As modalidades de tratamento prescritas são a utilização de drogas de uso local (tópico ou intralesional), ou sistêmico (oral ou injetável) e cirúrgico (PAGNONCELLI, 1994; METRY, 2000; ROCHA, 2000; TRUJILLO, 2008; BOYE, 2009; RAY, 2009).

O tratamento clínico medicamentoso inclui o uso de corticosteroides, interferon alfa e recentemente há a indicação de betabloqueadores (PUTTGEN, 2010). Estes medicamentos são dependentes de doses, duração e tempo de início do tratamento. A corticoterapia sistêmica está indicada especialmente em hemangiomas grandes ou agressivos. Os melhores efeitos da medicação são observados na fase proliferativa do tumor, ou seja, durante o primeiro ano de vida. A corticoterapia intralesional e tópica está indicada em lesões menores e de localização periocular. As infiltrações locais estão indicadas em intervalos de 4 a 6 semanas e o procedimento realizado em crianças sob anestesia geral ou sedação. Ainda sobre os corticosteroides, a prednisolona de uso sistêmico administrado por via oral tem sido largamente utilizada, porém com resposta terapêutica ainda variável. A dose dos protocolos varia de 1 a $3 \mathrm{mg} /$ $\mathrm{Kg} /$ dia, mantendo-se a medicação por 3 a 6 meses, se eficaz. A resposta é obtida na primeira semana do início do tratamento. Os efeitos adversos mais comumente relatados em relação ao uso da medicação são: a aparência cushingoide, irritabilidade, hipertensão arterial, transtornos gastrointestinais, diminuição da velocidade de crescimento do perímetro cefálico e ganho de peso, os quais são reversíveis com a suspensão da medicação. Em alguns casos, a suspensão da medicação pode causar efeito rebote e novo crescimento da lesão (PUTTGEN, 2010). Nos casos sem resposta efetiva ao corticosteroide, a opção mais frequentemente utilizada é o interferon (INF) alfa $2 \mathrm{a}$ e $2 \mathrm{~b}$, administrado por via subcutânea. $\mathrm{O}$ interferon alfa foi desenvolvido como agente antiviral. Esta medicação, apesar de bastante efetiva devido à inibição da movimentação do endotélio capilar in vitro, apresenta alta taxa de efeitos colaterais (febre, neutropenia e necrose de pele), o que requer atenção médica integral (ANGER, 1998; BALAU, 2007) A terapia medicamentosa com uso de IFN alfa, embora apresente um sucesso inicial, em casos de interrupção brusca do tratamento foi observada a recorrência imediata da lesão (ANGER, 1998). Desta forma, é necessária uma padronização para a retirada da medicação, baseada em testes sanguíneos. Embora ocorra a melhora dos sintomas clínicos, a anorexia e náusea também foram relatadas como efeitos colaterais da medicação. A recomendação a respeito da duração do tratamento é dependente da idade do paciente e severidade do tumor, sendo que a dificuldade reside em estabelecer o limite de crescimento tumoral. O tratamento deve ser mantido por um longo período de tempo até que a fase de maior risco de desenvolvimento do tumor acabe, mais ou menos em torno dos 6 aos 24 meses de idade. A partir daí, a remoção gradual da medicação está indicada. (ANGER, 1998; BALAU, 2007). O tratamento medicamentoso está indicado durante a fase proliferativa, sendo dependente da localização, tamanho e complicações associadas à lesão. Em relação aos hemangiomas de localização crítica, o uso de betabloqueadores (propanolol) está indicado como primeira linha de tratamento. (CAVALEIRO, 2011). $\mathrm{O}$ uso de betabloqueadores (propanolol) para o tratamento dos hemangiomas é recente, no entanto há dados que indicam a redução significativa do volume tumoral, mesmo em curto período de tempo e sugerem a presença de mecanismos de ação específicos. A dose inicial preconizada é de $1 \mathrm{mg} / \mathrm{Kg} / \mathrm{dia}$, de $8 / 8$ horas e aumentado para $2 \mathrm{mg} / \mathrm{Kg} /$ dia da quarta até a sexta dose, durante os primeiros seis meses de tratamento. (CAVALEIRO, 2011).

A escleroterapia no tratamento do hemangioma bucal está descrita com o uso da substância oleato de etanolamina a 5\% (EthamolinÒ), que causa total regressão da lesão por fibrose dos espaços endoteliais (PISTOIA, 1998). A glicose a $75 \%$, álcool a $95 \%$ e o 
polidocanol a 3\% também podem ser utilizados, mas em quantidades não superiores a $2 \mathrm{ml}$, com aplicações intralesional em intervalos de sete dias. A aplicação deve ser realizada no centro da lesão e profundamente para evitar a necrose tecidual (figuras 3 e 4 ). A escleroterapia está indicada em pacientes com vários anos de evolução da lesão e também como recurso prévio à cirurgia. (PISTOIA, 1998).

Figura 3 - Aplicação de esclerosante químico em hemangioma bucal.

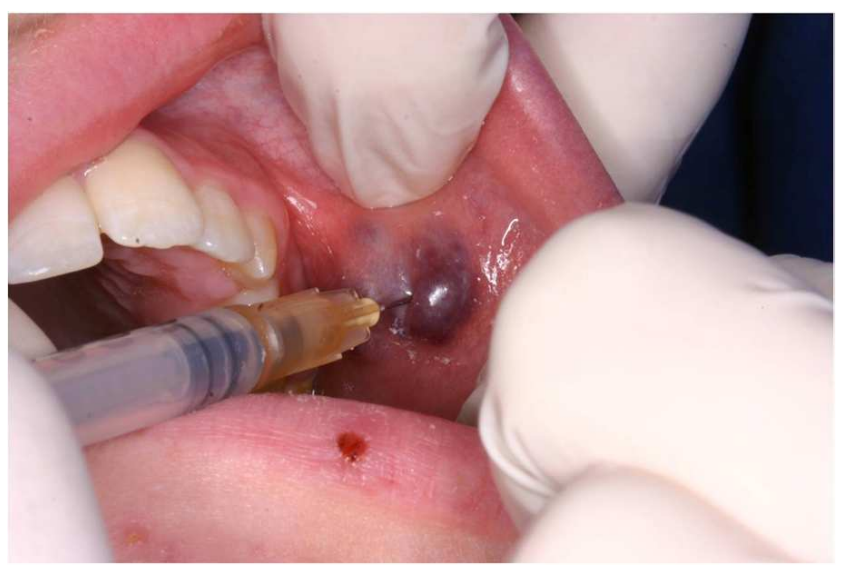

Figura 4 - Aspecto da lesão após sete dias de aplicação de esclerosante químico.

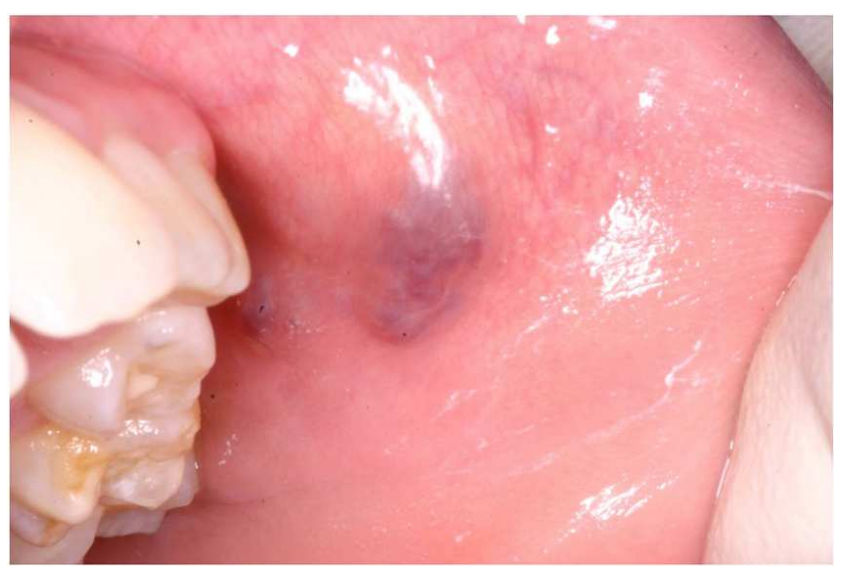

A cirurgia está indicada nos casos de emergência, ou ainda em lesões que não respondem ao tratamento sistêmico. O objetivo da ressecção cirúrgica é preservar funções essenciais como a visão, olfato e audição. Alguns autores relatam que o cirurgião deve orientar a ressecção precoce, com objetivo de prevenir traumas por estigma em crianças em fase pré-escolar (PITTA, 2009). A reparação cirúrgica em lesões residuais pós-tratamento está indicada somente aos 10 anos de idade que é quando o hemangioma já alcançou o ponto máximo de involução. $\mathrm{O}$ tratamen- to cirúrgico também é indicado para lesões em áreas com potencial desfigurante ou comprometimento funcional relativo como nariz, lábios e orelhas. Nestes casos, o crescimento do hemangioma pode causar distorção das estruturas em crescimento, e a ressecção pode contribuir para o desenvolvimento normal da região. O comprometimento estético e a dificuldade de convívio social na presença de lesões visíveis e estigmatizantes torna a indicação cirúrgica definitiva (PITTA, 2009). Nas lesões involuídas, o tratamento cirúrgico está indicado nas deformidades residuais definitivas, que não responderam à terapêutica medicamentosa. A ressecção permanente é indicada em hemangiomas profundos. As deformidades residuais encontradas são principalmente as telangiectasias, massas de tecidos fibrogordurosos e atrofia epidérmica.

Em hemangiomas de grande volume pode ser utilizado o Laser Nd: Yag (Laser Neodímio Granada de Alumínio Ítrio) e também a aplicação percutânea de crioterapia. No entanto, em hemangiomas de crescimento rápido mas de extensão limitada, é preconizada a realização da ressecção total, com objetivo de cessar a progressão da lesão. As indicações do uso de Laser que apresentaram bons resultados incluem, na fase proliferativa, os hemangiomas ulcerados e no tratamento de telangiectasias residuais, na fase involuída. As respostas positivas ao Laser ocorreram somente em lesões finas e superficiais, após múltiplas sessões (ULRICH, 2005).

Outros estudos indicam que hemangiomas não devem ser tratados e poderão regredir com nenhuma ou modesta cicatriz. Embora existam muitas modalidades terapêuticas efetivas, estes procedimentos raramente são capazes de recuperação da qualidade estética por completo. Os casos de insucesso do tratamento estão relacionados à presença de malformação vascular subjacente, que pode estar presente como fonte alimentadora do emaranhado vascular do hemangioma (figura 5 e 6). Nesta situação está indicada a avaliação pelo radiologista intervencionista para a possibilidade de embolização terapêutica da lesão (RICHTER, 2010). 
Figura 5 - hemangioma localizado próximo ao ângulo da mandíbula à esquerda.

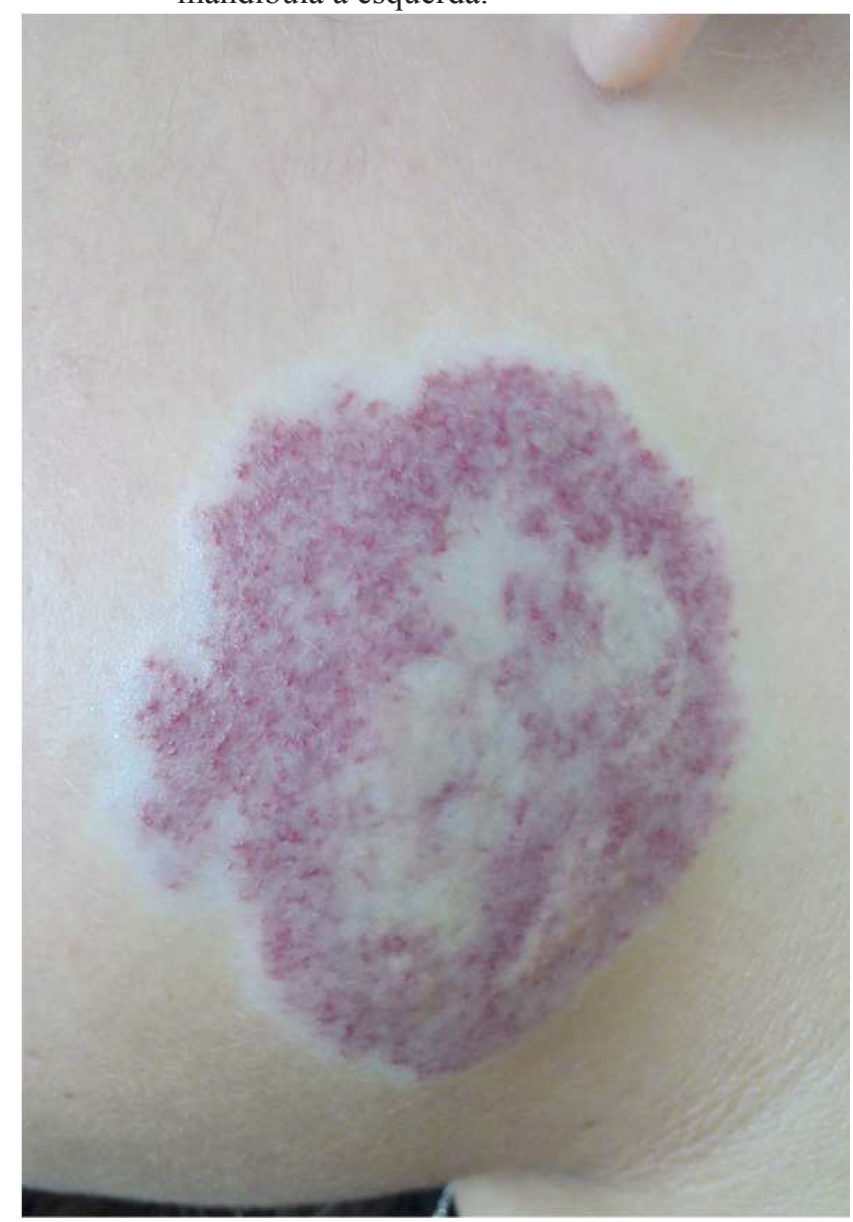

Figura 6 - Angiotomografia demonstrando associação de hemangioma e mal-formação? vascular em face.

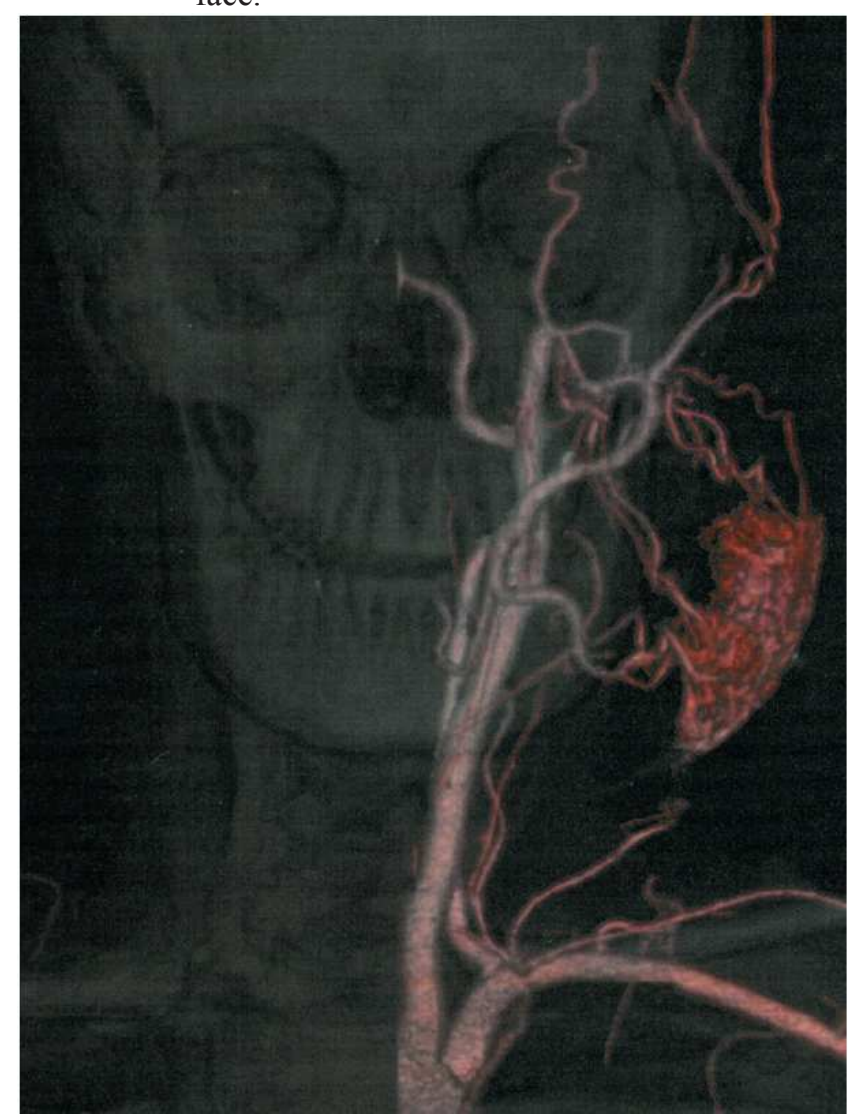

\section{CONCLUSÃO}

Um sistema racional de classificação é essencial para o manejo dos hemangiomas. A necessidade de avaliar com precisão a patogênese e o prognóstico é fundamental para o planejamento do tratamento. A filosofia de tratamento deve ser diferenciada em relação a critérios clínicos e características da lesão, como o tamanho, localização, evolução da lesão e idade do paciente. A identificação dos hemangiomas que necessitam de tratamento, o momento oportuno para o procedimento e a prevenção de implicações psicossociais do tumor sobre a criança e seus familiares são determinantes do sucesso do tratamento. A cautela é sempre necessária para o tratamento de pacientes portadores de anomalias vasculares.

O compromisso do cirurgião-dentista ao se deparar com paciente portador de hemangioma é direcionar e orientar, com a finalidade de prevenir as dificuldades decorrentes da presença da doença, a partir do desenvolvimento da criança. A abordagem multidisciplinar é direcionada para o enfoque 
integral do paciente pediátrico baseado na adequada comunicação profissional.

\section{REFERÊNCIAS}

AAlST, J. A. V.; BHUlleR, A.; SADOVES, A. M. Pediatric vascular lesions. The Journal of Craniofacial Surgery, n. 14, v. 4, p. 566-583, 2003.

ANGER, J.; CARNEIRO, R. G.; PINUS, J.; et al. The rebound effect in the treatment of complex hemangioma with interferon alpha 2A. Rev Paul Med, n. 116, v. 5, p. 1826-28, 1998.

BALAU, A. J.; DE NADAI, L. C.; BRESSAN, M. S.; SIMÃO, J. L. Tratamento de hemangioma gigante com interferon alfa: relato de dois casos. Rev Bras Hematol Hemoter, n. 29, v. 4, p. 406-411, 2007.

BOYE, E.; JINNIN, M.; OLSEN, B. R. Infantile hemangioma: challenges, new insights, and therapeutic promise. J Craniofac Surg, n. 20, suppl 1, p. 678-84, 2009.

CAVALEIRO, L. H. S; VIANA, F. O.; UNGER, D. A. A. et al. Hemangiomas extensos da infância tratados com propranolol: relato de dois casos. J Vasc Brás, n. 10, v. 2, p. 173-176, 2011.

DELLA NINA, B. I.; OLIVEIRA, Z. N. P.; MACHADO, M. C. M. R.; MÁCEA, J. M. Apresentação, evolução e tratamento dos hemangiomas cutâneos - experiência do ambulatório de Dermatologia Infantil do Hospital de Clinicas da Faculdade de Medicina da Universidade de São Paulo. An Bras Dermatol, n. 81, v. 4, p. 323-7, 2006.

DILSIZ, A.; AYDIN, T.; GURSAN, N. Capillary hemangioma as a rare benign tumor of the oral cavity: a case report. Cases J, n. 2, p. 8622, 2009.

ELIAS, R.; WEIGERT, K. L.; FIGUEIREDO, M. A. Z.; YURGEL, L. S. Hemangioma, granuloma piogênico ou sarcoma de Kaposi? Diagnóstico diferencial em estomatologia. Revista Brasileira de Odontologia. 2004; 61(2): 84-87.

ERNEMANN, U; KRAMER, U; MILLER, S; et al. Current concepts in the classification, diagnosis and treatment of vascular anomalies. European Journal of Radiology, n. 75, v. 1, p. 2-11, 2010.

ETHUMANDAM, M.; MELLOR, T. K. Haemangiomas and vascular malformations of the maxillofacial region - a review. British Journal of Oral and Maxillofacial Surgery, n. 44, v. 4, p. 263-272, 2006.

GONTIJO, B.; SILVA, C. M. R.; PEREIRA, L. B. Hemangioma da infância. An Bras Dermatol, n. 78, v. 6, p. 651-673, 2003.
METRY, D. W.; HEBERT, A. A. Benign cutaneous vascular tumors of infancy: when to worry, what to do. Arch Dermatol, n. 136, v. 7, p. 905-14, 2000.

MULLIKEN, J. B.; GLOWACKI, J. Hemangiomas and vascular malformations in infants and children: a classification based on endothelial characteristics. Plast Reconstr Surg, n. 69, v. 3, p. 412-22, 1982.

PAGNONCELLI, R. M.; FILHO, M. S. Tratamento de hemangioma capilar - relato de caso. R. Fac. Odontol, n. 35 , v. 1, p. 2-5, 1994.

PISTOIA, A. D. Uso de agente de esclerose química para o tratamento de hemangioma - relato de caso. Revista Odonto Ciência, n. 1, v. 25, p. 39-48, 1998.

PITTA, G. B. B.; GOMES, R. R. Tratamento de hemangioma ulcerado: relato de caso. J Vasc Brás, n. 8, v. 3, p. 263-266, 2009

PUTTGEN, K. B.; PEARL, M.; TEKES, A.; MITCHELL, S. E. Update on pediatric extracranial vascular anomalies of the head and neck. Child Nerv Syste, n. 26, v. 10, p. 14171433,2010 .

RAY, B. W.; MATTHEW, I. R. How do I manage a suspected oral vascular malformation? Journal Canadian Dental Association, n. 75, v. 8, p. 575-577, 2009.

RICHTER, G. T.; FRIEDMAN, A. B. Hemangiomas and Vascular Malformations: Current Theory and Management. International Journal of Pediatrics, p. 1-10. Article ID 645678, 2010.

ROCHA, L. B.; PÁDUA, J. M.; MARTINS, R. H.; LIA, R. C. C. Hemangioma da cavidade bucal. Rev Gau de Odontol, n. 48, v. 3, p. 150-152, 2000.

SILVA, F. M.; PORTOLAN, F. B. A. M.; FIQUEIREDO, P. J. Hemangioma. Rev Fac Odontol Lins, n. 12, p. 57-59, 2000.

TRUJILLO, B. Manejo de lesiones vasculares benignas. Gaceta Médica de Caracas, n. 116, v. 2, p. 134-142, 2008.

ULRICH, H.; BÄUMLER, W.; HOHENLEUTNER, U.; LANDTHALER, M. Neodymium-YAG Laser for hemangiomas and vascular malformations -- long term results. J Dtsch Dermatol Ges, n. 3, v. 6, p. 436-40, 2005. 